\title{
Extending WiMAX to New Scenarios: Key Results on System Architecture and Test-beds of the WEIRD project
}

\author{
INVITED PAPER
}

\author{
G. Martufi \\ Elsag Datamat \\ Italy
}

giuseppe.martufi@elsagdatamat.com

\section{Curado}

University of Coimbra

Portugal

marilia@dei.uc.pt

$$
\begin{aligned}
& \text { E. Piri } \\
& \text { VTT } \\
& \text { Finland }
\end{aligned}
$$

esa.piri@vtt.fi

\author{
M. Katz \\ VTT \\ Finland \\ marcos.katz@vtt.fi \\ M. Castrucci \\ University of Rome "Sapienza" \\ Italy \\ marco.castrucci@dis.uniroma1.it
}

\author{
P. Neves \\ Portugal Telecom Inovaçaò \\ Portugal \\ est-p-neves@ptinovacao.pt \\ P. Simoes \\ University of Coimbra \\ Portugal \\ psimoes@dei.uc.pt \\ K. Pentikousis \\ VTT \\ Finland \\ kostas.pentikousis@vtt.fi
}

\begin{abstract}
Wireless Metropolitan Area Networks based on IEEE 802.16 standards are in the deployment phase in many countries. In fact, this technology can deliver high data rate while covering long distances, being a suitable candidate to solve the problem of digital divide and thus being able to provide connectivity in isolated and impervious areas. This paper presents the results of the EU Integrated Project WEIRD (WiMAX Extension to Isolated Research Data networks), in which WiMAX technology has been used to provide connectivity in impervious areas to perform volcano monitoring, fire prevention and telemedicine. In particular, we describe the advantages of the WEIRD system architecture and we show how WiMAX test-beds have been set up within the project to demonstrate the possibility to use WiMAX to extend the connectivity of the pan-European data communication network GEANT2 to stringent scenarios.
\end{abstract}

\section{Categories and Subject Descriptors}

C2.1 [Computer - Communication Networks]: Network architectures and design - networks communications, wireless communication.

\section{General Terms}

Design, Experimentation.
Keywords WEIRD, WiMAX, test-beds, QoS.

\section{INTRODUCTION}

The WEIRD Project [23] aims to develop an infrastructure filling the gap between broadband network and applications: one of the main outcomes of this project is a service allocation and signalling system allowing the enforcement of QoS resource reservation policies into underlying broadband networks. In fact WEIRD infrastructure [3] is able to map application QoS requirements making the most of the features available in each broadband access technology, especially wireless.

From a social point of view, WEIRD helps to overcome the geographical discrimination due to the broadband service availability and as a consequence, encourages the growth of new business activities. WEIRD proposes a support technology extremely useful in those scenarios where human presence cannot be continuously granted or moving is not easy. These novel scenarios include the monitoring of remote or hazardous areas, such as a volcanic sites monitoring, fire prevention, or the communication between isolated spots.

By adopting the special software capabilities provided by WEIRD, broadband networks can better support next generation services. In fact, the proposed approach has stimulated the interest of user-communities working with resource-demanding applications, such as: Fire Prevention, Telemedicine and Environmental Monitoring. These communities have actively participated in the project and the benefits gained by the usage of specific applications in those areas together with WEIRD software have been validated by several trials. Thus the WEIRD project promotes solutions for scientific and industrial applications proved in large scale experimentation involving real settings.
Mobimedia 2008 July 7-9, 2008, Oulu, Finland. Copyright 2008 ICST ISBN 978-963-9799-25-7 DOI 10.4108/ICST.MOBIMEDIA2008.3833 
WEIRD project proposes broadband connectivity based on WiMAX, a wireless technology providing a flexible, costeffective, standards-based means of enhancing broadband services not envisioned in a "wired" world. It also facilitates interoperability across heterogeneous technology domains focusing on wireless technologies belonging to the state-of-theart. This research project is also complementary to and in support of the extension of GÉANT, the Pan-European Gigabit Research and Education Network, by interconnecting the National Research Networks with Broadband Wireless connected test-beds. The proposed approach allows an NRN belonging to a participating country or operating in impervious environments to easily connect isolated research communities to the GÉANT network. The outcomes of WEIRD have been developed according to models and protocols from major standardization bodies and fora.

The paper is organized as follow: section 2 describes the WEIRD system architecture and highlights its main features; section 3 shows some of the application scenarios that benefit from the WEIRD system while in section 4 the WEIRD project test-beds are described, together with some collected results. Finally, section 5 draws the conclusions.

\section{WEIRD ARCHITECTURE}

The IEEE 802.16 standard [7][8] does not specify an End-to-End (E2E) system, but rather defines only the Physical (PHY) and the Medium Access Control (MAC) layers of the architectural stack. The WiMAX Forum [24][25] is currently extending the IEEE 802.16 architecture, by defining the Network Reference Model (NRM). The NRM is a logical representation of the WiMAX network and its main goal is to guarantee interoperability between distinct WiMAX products. The WEIRD control plane architecture, illustrated in Figure 1, is fully compliant with the
WiMAX Forum guidelines, in particular with the NRM. It is composed by a set of standardized interfaces, also known as WiMAX Reference Points (WRPs), and by three functional entities:

1. Connectivity Service Network (CSN) which contains the core network entities, such as, the DHCP, DNS, AAA and SIP servers, and establish connectivity with the IP backbone;

2. Access Service Network (ASN) that is composed by several WiMAX BSs connected to multiple ASN-GWs (ASN Gateways), which are responsible to establish connectivity with the CSN. The ASN includes the required functionalities to provide radio connectivity to the WiMAX subscribers;

3. Mobile Station (MS): terminal equipment, responsible to establish radio connectivity with the WiMAX BS.

WEIRD is designed to support a wide range of applications, from legacy applications which are not capable of explicitly requesting any type of QoS, to WEIRD aware applications (e.g., SIP [22]) which can profit from the QoS support provided by the WEIRD system. Multimedia communication applications, such as audio and video, rely on SIP for session control. In the WEIRD system, resource reservation for this type of applications is also triggered by a QoS aware version of SIP. Concerning the signalling for non-SIP applications, two situations are considered. In the first case, the application can be modified in order to interact with the WEIRD system through the WEIRD API, in order to trigger resource reservation and other WEIRD services such as AAA functions.

In the second case, for legacy applications, it is not possible to modify the application, but WEIRD services can be accessible through an agent, called the WEIRD Agent, which can be either off-line or on-line.

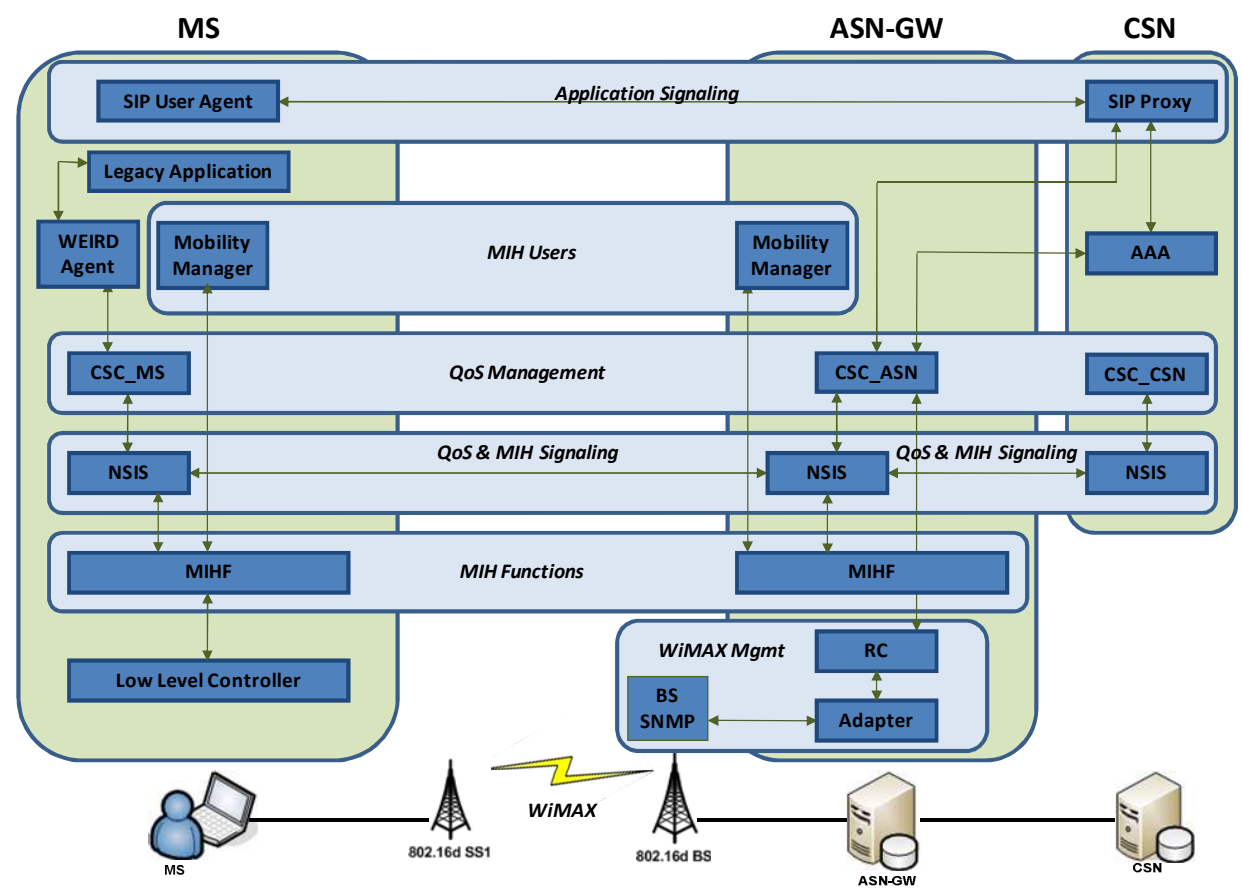

Figure 1. WEIRD architecture overview 
The off-line WEIRD Agent is employed by the end-user to manually allocate the resources before the application starts. The on-line WEIRD Agent detects flows from known application and automatically triggers the resource allocation needed for that type of application. With these agents, applications which are not aware of the WEIRD system may take advantage of its capabilities, without the need to be modified.

In order to guarantee QoS on the access network, and fully integrate the WiMAX inherent QoS capabilities, WEIRD has specified and implemented a Connectivity Service Controller ( CSC module. The CSC module is the core QoS component, distributed across the three functional entities of the WiMAX NRM: Connectivity Service Controller in the CSN ( $\left.C S C_{-} C S N\right)$, Connectivity Service Controller in the ASN (CSC_ASN) and Connectivity Service Controller in MS (CSC_MS). The $\boldsymbol{C S C} \boldsymbol{A} \boldsymbol{A N}$ is the main QoS coordination point, being responsible for the interaction with the SIP Proxy and the AAA servers, as well as with the NSIS QoS signalling protocol. Furthermore, the $\boldsymbol{C S C} \boldsymbol{C A S N}_{\mathbf{N}}$ is also responsible, through the interaction with the Resource Controller $(\boldsymbol{R C})$, for admission control and resources allocation in the WiMAX segment. The $\boldsymbol{C S C}_{-} \boldsymbol{C S N}$ is responsible to establish the QoS reservations towards the core network, whereas the $\boldsymbol{C S C} \boldsymbol{M} \boldsymbol{S}$, using an interface with the WEIRD Agent, is responsible for integrating legacy applications within the WEIRD system.

Next Steps in Signalling (NSIS) is a new framework being developed in the NSIS Working Group of the IETF [21]. This group is responsible for the standardization of an IP signalling protocol suite, being QoS signalling the first application. The NSIS framework is composed by two layers, namely the NSIS Transport Layer Protocol (NTLP) and the NSIS Signalling Layer Protocol (NSLP). The modularity of the framework, with the separation of the transport of the signalling messages from the signalling application, allows for an easy extension to support new applications which take advantage of the transport mechanisms of NSIS. The NTLP is also named General Internet Signalling Transport (GIST), and is in charge of the transport of signalling messages through the network. The NSLP contains the functionalities of the different signalling applications, namely the Quality of Service NSLP (QoS-NSLP), the Network Address Translation (NAT) and Firewall (FW) NSLP, among others being currently developed [18].

The NSIS framework is used in WEIRD to achieve end-to-end QoS signalling. QoS-NSLP is used to signal the QoS requirements of the different types of applications throughout the network in order to trigger the mechanisms of resource allocation along the path to the destination. The NSIS Working Group specifies the QoS Specification (QSPEC) object for the encapsulation of the QoS parameters [6].

Table 1. - Mapping between the WiMAX and differentiated services QoS model

\begin{tabular}{|c|c|}
\hline WiMAX & Differentiated Services \\
\hline UGS & EF \\
\hline rtPS & AF3 \\
\hline nrtPS & AF2 \\
\hline BE & BE \\
\hline
\end{tabular}

WEIRD has extended the generic QSPEC object to include specific QoS parameters of WiMAX, such as Minimum Reserved Traffic Rate, Maximum Sustained Traffic Rate, Scheduling Class, Unsolicited Grant Interval, Minimum Latency and Tolerated Jitter. In this context, in order to achieve end-to-end QoS signalling between domains with different QoS models, that is, where QoS levels are characterized with a different set of QoS parameters, a mapping between the WEIRD/WiMAX QoS Model and the Differentiated Services QoS Model used in the core network was conceived, as shown in Table 1 .

As previously mentioned, in WEIRD, the GIST and QoS-NSLP components of NSIS are used for the QoS signalling between the CSCs of the WEIRD system, namely the CSC_MS, CSC_ASN and $C_{S C}$ CSN. The resource reservation is triggered at the CSC_ASN, which is signalled from two different sources, depending on the type of application to be used, namely, legacy or SIP aware applications. In the first case, the QoS signalling request that arrives at the $\boldsymbol{C S C} \boldsymbol{A}_{-} \boldsymbol{A S N}$ is sent by the $\boldsymbol{C S C} \boldsymbol{M} \boldsymbol{M}$. In the second case, the QoS signalling request is sent by the SIP Proxy. In both situations, the QoS-NSLP is used to depict the information needed for the resource allocation on the WiMAX link and to notify all the entities of the QoS-enabled domains on the end-to-end path. To optimize the mobility procedures, WEIRD has integrated the IEEE 802.21 [9] framework. As illustrated in Figure 1, the 802.21 MIHF modules are distributed across the several entities of the NRM, providing link layer events and commands to the registered MIHUs. The Mobility Manager at the MS (MM@MS) is responsible for Mobile Initiated Handover (MIHO) decisions, whereas the $\boldsymbol{M M} @ \boldsymbol{A S N}$ is responsible for Network Initiated Handover (NIHO) decisions. To interface with the link layer we have defined the Low Level Controller (LLC), which interacts with the MIHF using a standardized MIH_LINK_SAP interface. The MM is also responsible to establish the interaction with the QoS entities, enabling makebefore-break scenarios.

All functions related with the WiMAX system are managed and controlled by the $\boldsymbol{R C}$, which can be seen as the WiMAX link manager. The $\boldsymbol{R C}$ is responsible for the QoS management in the WiMAX link, including Service Flows and Convergence Sublayer classifiers control. To enforce the QoS decisions on the WiMAX $\mathrm{BS}$, the $R C$ triggers the Adapter module that will communicate the decisions to the WiMAX BS through an SNMP [12] interface. It is split into a Generic Adapter (GA) component and one or more Vendor-Specific Adapter (VSA) modules. With this approach, different WiMAX equipment can be integrated into the WEIRD architecture by developing a VSA for the particular equipment of each vendor.

\section{APPLICATION SCENARIOS}

Due to the features offered by WiMAX technology to reach high data rate over long distances, the WEIRD system is very useful in scenarios of difficult access, or where human presence cannot be continuously granted. These scenarios include the monitoring of remote or dangerous areas, such as a volcanic sites monitoring, fire prevention, or the support for emergencies application like telemedicine. In particular, the WEIRD system has been designed and tested to meet requirements imposed by the aforementioned scenarios. In the following subsections we describe in detail some of the scenarios that can benefit from WEIRD system capabilities. 


\subsection{Telemedicine}

E-Health is already playing a key role in many countries in making healthcare a more accessible, high-quality, cost-effective, sustainable and safe service for citizens. Figure 2 shows an example of telemedicine application. Some examples of advanced medical service that require broadband wireless data connection are:

- Remote follow-up and assistance: often, after therapies or surgical procedures, patients need to be monitored and assisted by medical personnel. Up to now, patients have to travel to far-away hospitals, even from isolated and rural areas to be visited by doctors. In this case, the use of the WEIRD system can be very useful to allow remote follow-up and assistance of patients, by establishing voice and video over IP connections to support real-time communications with QoS assurance between the patient and the doctor.

- Remote diagnosis and need to intervene on non transportable patients: in case of childbirth at home, street accidents or other emergencies, there is the need to transmit urgently critical data in order to make an immediate diagnosis. In this case, WEIRD system can be used to provide connectivity to doctors in the field where usually there is the lack of broadband access networks. Thus, voice and video over IP connections are established to transmit real-time information with an adequate level of QoS in both fixed (from the accident place) and mobile (from the ambulance going to the hospital) situations.

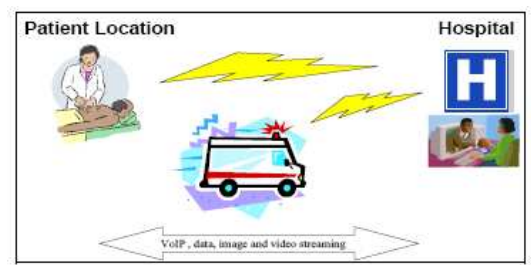

Figure 2. Medical information exchange from patient location or while travelling

\subsection{Volcano monitoring}

Active volcanoes represent on of the highest natural risk in the world, as volcano eruptions can cause heavy damages to house and facilities, as well as the death of people living in volcanic areas. A constant monitoring of volcanic activity can be useful to foresee possible eruptions and then undertake the best strategy to reduce the disruptive effects of such events. Nowadays, seismology is the most useful method to monitor volcanic activity, as a large variety of different seismic signals occur before an eruption. As an example of volcano monitoring, we report the activity realized by the Osservatorio Vesuviano, a department of Istituto Nazionale di Geofisica e Vulcanologia (INGV-OV, Italy), which is in charge of continuous monitoring of the Neapolitan active volcanoes (Vesuvius, Campi Flegrei and Ischia Island) [2].

A real-time seismic monitoring is performed thanks to a Permanent Seismic Network realized with sensors that collect data continuously. But such data is insufficient to study in detail the seismic activity during a crisis. During these events, a Temporary Seismic Network is deployed on the volcanic area in order to extend the permanent one. Such a temporary network is composed by portable digital seismic stations with local recording on removal media that needs a periodic check to retrieve data and thus it does not allow a real-time integration with permanent network data.

The WEIRD system can be used in this scenario in order to provide a wireless connection to be used to transmit data collected by the temporary network to the Acquisition Centre where scientists analyze received information. This solution allows a real-time evaluation of data and, in addition, it avoids the need for operators to reach every station to download collected data. Moreover, WiMAX connectivity offered by the WEIRD system can also be used in critical situations to offer voice communication between the Acquisition Centre and the teams of scientists involved in field activities.

\subsection{Fire prevention}

Climatic changes and new land use practices have turned forest fires into a major disaster in extensive areas of southern Europe. In addition to the dramatic loss of lives and property, forest fires also increase the risk of desertification and the emission of greenhouse gases.

In spite of considerable public investments, fire prevention and fire fight efforts are still largely unsuccessful. The difficulty to guarantee early detection of the fire has been one of the main causes of uncontrolled fire spread. This is especially true in areas which are scarcely populated or difficult to reach. In these areas, fire detection is performed by human spotters - based on surveillance towers or, in some cases, small airplanes - searching for smoke and communicating their findings to centralized control centres. These methods are not cost-efficient: each tower requires the permanent presence of human watchers, while airplane patrols become even more expensive. Moreover, verbal radio communication can be ineffective as the control centre may lose precious minutes receiving the spotter description and precisely locating the potential fire.

Communication between surveillance towers and control centres is therefore one of the problems within this scenario. Broadband technologies such as ADSL or 3G/UMTS are not available in remote forests. Alternatives like GPRS, GSM or UHF radio-links, besides also presenting coverage limitations, cannot provide the bandwidth required for more automated fire detection.

WiMAX appears to fit well in this scenario. Given its potential range, bandwidth and adaptability to environmental conditions, WiMAX may provide connectivity to remote monitoring systems, capable of effectively providing early detection of fire in a more efficient and cost-effective manner. Cameras mounted in surveillance towers can automatically scan a 360 degrees area, with $20-40 \mathrm{~km}$ radius, and send collected data (live video streaming, infrared sensors, automated pre-detection of potential fires, location data, meteorological data) to control centres, where prospective fires are further investigated (for instance remotely pointing and zooming the cameras to the suspect area). This solution is both cost-effective (the number of required human operators is drastically reduced) and efficient (data received by the control centre is more precise and can lead to instant location of the fire, by triangulation and association with geographical information systems). 
During fire fighting, WiMAX may also provide better communication between involved partners, for instance allowing the instant exchange of data between fire-fighters and the control centre (GPS-location of the fire-fighters, meteorological data, images of the fire, instructions from the control centre, etc.)

\section{WEIRD TEST-BEDS}

This Section presents a short description of two of the test-beds developed in the WEIRD Project. A more extensive description of the test-beds developed in the context of the WEIRD Project, as well as related results, may be found at [5].

\subsection{Coimbra test-bed description and results}

In order to assess the suitability of WiMAX for the already discussed fire prevention scenario, a simple test-bed was set up in Coimbra, Portugal, encompassing two fire surveillance towers (Serra da Lousã and Serra do Carvalho) already in use by regional fire prevention services. Each of these towers is now equipped with surveillance cameras, digital compasses (for geographical location of detected fires), and weather stations (wind, temperature, humidity). Collected data feeds an application located in a control centre, making it possible to immediately pinpoint potential fires in geographical information databases (allowing better guidance of the first response fire fighters, transported by helicopter) and to save video and data for forensic analysis (e.g. criminal or scientific investigation)

Figure 3 presents the topology of the test-bed. A $22 \mathrm{~km} \mathrm{WiMAX}$ link connects the University of Coimbra with the first surveillance unit (Serra da Lousã). A second WiMAX link connects Serra da Lousã with Serra do Carvalho (19 km), further stretching WiMAX coverage into the remote forest. Both links are point-tomultipoint, making it possible to add further surveillance units, in the future.

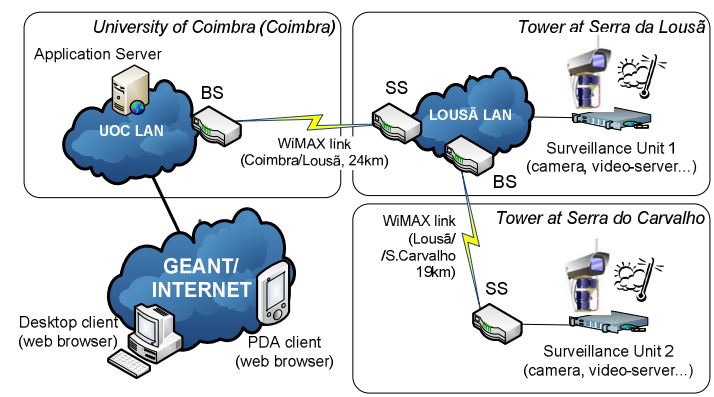

Figure 3. Fire prevention test-bed

So far tests on the fire prevention test-bed focused on functional issues and the validation of the QoS mechanisms of the WEIRD architecture. The system is currently using the $3443-3471 \mathrm{MHz}$ range for one of the links and the $3543-3571 \mathrm{MHz}$ range for the other. Table 2 presents the basic configuration of each Base Station and Subscriber Station of the test-bed. Several tests were conducted using $3.5 \mathrm{MHz}, 7 \mathrm{MHz}$ and $14 \mathrm{MHz}$ channels, in order to assess the maximum throughput of each channel.

Table 2. Fire prevention test-bed configuration

\begin{tabular}{|l|l|c|c|}
\hline Unit & BW (MHz) & Power $\mathbf{( d B m )}$ & Antenna \\
\hline BS (Coimbra) & $3.5,7$ and 14 & 23 & $90^{\circ}$ \\
\hline SS (Lousã) & $3.5,7$ and 14 & 16 & $15^{\circ}$ \\
\hline
\end{tabular}

\begin{tabular}{|l|c|c|c|}
\hline BS (Lousã) & $3.5,7$ and 14 & 23 & $90^{\circ}$ \\
\hline SS (Carvalho) & $3.5,7$ and 14 & 16 & $15^{\circ}$ \\
\hline
\end{tabular}

Tests were conducted in each WiMAX link, using IPerf to measure the maximum bandwidth available in each link. The results for the weakest link (Coimbra/Lousã), are presented in Table 3, showing that the available bandwidth is enough for the traffic generated by the surveillance units (around $1.5 \mathrm{Mbps}$ per surveillance unit), even without using any sort of aggressive compression mechanisms or pre-processing of the video streams. These results - affected by the distance involved and not-perfect LOS conditions - could be extensively improved by better antenna alignment or the use of better antennas. Measured results for the second link (Lousã/Carvalho) were significantly better, due to the slightly shorter distance and the much better LOS conditions.

Table 3. IPerf measurements (Coimbra / Lousã)

\begin{tabular}{|c|c|c|c|}
\hline Channel & Uplink (Mbps) & Downlink(Mbps) & Total(Mbps) \\
\hline $3.5 \mathrm{MHz}$ & 1.1 & 1.3 & 2.4 \\
\hline $7.0 \mathrm{MHz}$ & 2.2 & 2.7 & 4.9 \\
\hline $14.0 \mathrm{MHz}$ & 4.5 & 5.6 & 10.1 \\
\hline
\end{tabular}

End-user tests have been informal, based on the comparison between the current procedures - human spotter located in the same towers, manually detecting the fires and verbally communicating with the control centre - and the new infrastructure. More complete evaluation studies will take place during the next fire season (from June/2008 to September/2008). Support for mobile nodes (such as laptops or PDAs installed in the fire-fighter vehicles) was also integrated in the fire prevention platform and will be tested subsequently.

Another important set of tests relates with the validation of the proposed QoS management architecture. In this context, several tests were conducted, focusing in the end-to-end processing overhead introduced by the QoS signaling integrated in the WEIRD system. Measured results show that it this overhead is within acceptable bounds, with an impact in the overall system likely lower than application level signaling such as the SIP. A detailed discussion of performance of the WEIRD QoS signaling framework is provided at [1].

\subsection{Oulu test-bed description and results}

In the framework of the WEIRD project, VTT has deployed a fixed WiMAX test-bed using Airspan equipment and is finalizing deployment of a mobile WiMAX test-bed based on AlcatelLucent equipment. This subsection overviews the WiMAX testbeds at VTT and summarizes recent results from empirical investigations using real WiMAX equipment. Figure 4 illustrates the VTT fixed WiMAX test-bed used in our empirical performance evaluation studies. The WiMAX equipment in the test-bed consists of Airspan MicroMAX-SoC base station (BS) and Airspan ProST and EasyST subscriber stations (SS). Equipment resides in an indoor laboratory with short distances which explains the low transmitting power. As shown in Figure 4, GNU/Linux PCs are symmetrically connected on the BS and SS side to act as traffic sources/sinks. We have recently evaluated the performance of fixed WiMAX equipment with live IPTV streams 
and Jugi's Traffic Generator (JTG) [13] used both to inject synthetic VoIP packets and reproduced IPTV streams into the test-bed. Synchronization of the end hosts is in the order of tens of microseconds, and is achieved by employing PTPd [14], an open source implementation of IEEE 1588 Precision Time Prototocol (PTP) [11]. The accuracy of PTPd when used over Ethernet is similar to GPS-based synchronization, as studied in [17]. The testbed is fully IPv6 compatible and can be connected directly to the GEANT network.

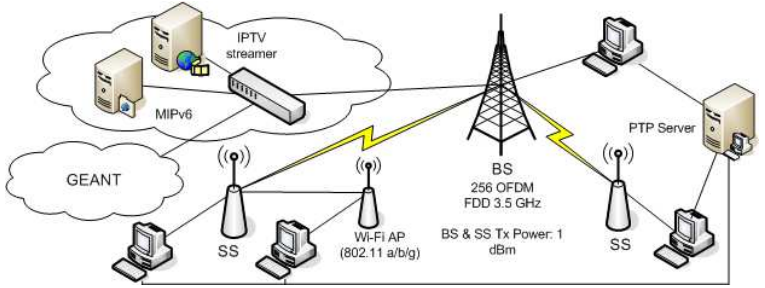

Figure 4. Schematic of the Oulu fixed WiMAX test-bed

As noted in [15], there are very little empirical studies of what actually can be done with WiMAX in practice today. With Airspan equipment we have obtained with maximum transmission unit (MTU) of 1500 bytes and UDP goodput levels for uplink $5.515 \mathrm{Mb} / \mathrm{s}$ and for downlink $9.395 \mathrm{Mb} / \mathrm{s}$. With TCP and same MTU size we have recorded goodputs of $5.417 \mathrm{Mb} / \mathrm{s}$ and 9.213 $\mathrm{Mb} / \mathrm{s}$ for the uplink and downlink, respectively. However, such capacity utilization level cannot be attained when the WiMAX access network is swamped with small packets. As detailed in [15], the small size of single-sample G.723.1 VoIP packets (24 bytes of application payload) has a substantial effect on the cumulative goodput and the number of flows that can be supported on the uplink and downlink of our WiMAX testbed. Injecting only G.723.1 VoIP flows into the fixed WiMAX link saturates the cumulative goodput to $1.5 \mathrm{Mbits} / \mathrm{s}$ in uplink. In downlink, the corresponding saturation point is $3.3 \mathrm{Mb} / \mathrm{s}$ only. Using simple application-layer aggregation scheme to bundle multiple voice frame samples into one packet increases the cumulative goodput saturation point significantly. Aggregation of three voice frame samples at application-layer nearly doubles the cumulative goodput capacity. It effectively more than triples the sustained good quality (taking into account sample loss and oneway end-to-end delay) VoIP flow amount compared to a nonaggregated scheme. With this aggregation level, VoIP packet is generated every $90 \mathrm{~ms}$ which is still acceptable delay increase in most of the cases.

VoIP aggregation, carried out at network-layer bundling multiple VoIP IP-packets into one injected VoIP packet, does not attain any enhancement in terms of cumulative goodput. However, aggregation of three VoIP packets at network-layer more than doubles the sustained VoIP flow amount. In addition to VoIP aggregation, another way to reduce the significant header overhead produced by RTP/UDP/IP in VoIP traffic is to use Robust Header Compression (ROHC). It cuts the total RTP/UDP/IP header size to few bytes only in most of the packets. As found in [4], the most significant benefit from ROHC was achieved when used with aggregated VoIP packets. In case of two voice sample frames in one packet, ROHC improves the total sustained G.729.1 bi-directional VoIP flow amount by 29\% compared to uncompressed aggregated VoIP flows. Basically, the emphasis is on the fact that small packets seem to cause clear underutilization on the specific WiMAX equipment. Table 4 summarizes the effect of different MTU sizes to the total downlink throughput. Employed modulation scheme is 64QAM $(\mathrm{FEC}=3 / 4)$. As reported in [19], these throughput levels can be achieved, to some extent, at a distance of up to $5 \mathrm{~km}$ from BS even though these measurements are performed in a laboratory environment. In [16] we have compared the performance of WiMAX and Wi-Fi extended WiMAX (as illustrated in Figure 4) with VoIP and IPTV streams. We found out that Wi-Fi clearly underperforms for VoIP compared to WiMAX despite the significant difference in nominal capacities.

VTT also carried out studies on the impact of the new scenarios to WiMAX performance, from a physical layer perspective. A new channel model for mountainous areas was developed and tested [20]. Results show that in real mountainous environment the delay spread could greatly exceed the margin given by the cyclic prefix, deteriorating significantly the performance. the use of multiantenna techniques can effectively combat the detrimental effects of such scenarios, as shown in [10].

Table 4. Throughput comparison with different MTU sizes

\begin{tabular}{|l|l|l|l|}
\cline { 2 - 4 } \multicolumn{1}{c|}{} & $1500 \mathrm{~B}$ & $112 \mathrm{~B}$ & $64 \mathrm{~B}$ \\
\hline $\mathrm{DL}$ & $9.6 \mathrm{MB} / \mathrm{s}$ & $9.2 \mathrm{MB} / \mathrm{s}$ & $8.8 \mathrm{MB} / \mathrm{s}$ \\
\hline $\mathrm{UL}$ & $5.6 \mathrm{MB} / \mathrm{s}$ & $5.4 \mathrm{MB} / \mathrm{s}$ & $4.0 \mathrm{MB} / \mathrm{s}$ \\
\hline
\end{tabular}

VTT's mobile WiMAX test-bed consists of Alcatel-Lucent equipment comprising two 9116 BSs and other vendor-specific necessary radio access network (RAN) equipment. Moreover, testbed includes $802.16 \mathrm{e}$ PCMCIA cards and indoor and outdoor customer premise equipment (CPE). The test-bed operates in the $3.5 \mathrm{GHz}$ band and currently employs $5 \mathrm{MHz}$ bandwidth. VTT's mobile WiMAX employs Time-Division Duplex (TDD). BS transmitting power can be configured from $25 \mathrm{dBm}$ to $35 \mathrm{dBm}$.

\section{CONCLUSIONS}

In this paper we present an overview of the WEIRD project and its key results. The goal of the project has been to extend WiMAX systems to novel scenarios, and this has been carried out from many perspectives. The WEIRD Architecture is first discussed, always taking into account the provision of end-to-end QoS. The novel WEIRD scenarios are also presented and discussed, particularly telemedicine, volcano monitoring and fire prevention. Several WEIRD test-beds were deployed in Europe, and in this paper we described the ones deployed in Coimbra (Portugal) and Oulu (Finland). Test-beds were associated with particular scenarios, with the former corresponding mostly to fire prevention cases, whereas the task of the latter was the delivery of multimedia signal as well as volcano monitoring. Some performance results measured on the real test-beds are also included, though extensive results can be found in the project deliverables and published papers.

\section{ACKNOWLEDGMENTS}

The authors would like to thank the contributions of all members of the WEIRD team.

\section{DISCLAIMER}

The work described in this paper is based on result of IST FP6 Integrated Project WEIRD. WEIRD receives research funding 
from the European Community's Sixth Framework Programme. Apart from this, the European Commission has no responsibility for the content of this paper. The information in this document is provided as is and no guarantee or warranty is given that the information is fit for any particular purpose. The user thereof uses the information at its sole risk and liability.

\section{REFERENCES}

[1] B. Sousa et al., "The Cost of Using IEEE 802.16d Dynamic Channel Configuration", Proceedings of the 2008 IEEE Int. Conf. on Communications (ICC 2008), Beijing, May 2008

[2] Castellano M., Buonocunto C., Capello M., and M. La Rocca, "Seismic surveillance of active volcanoes: the Osservatorio Vesuviano Seismic Network (OVSN - Southern Italy)", Seism. Res. Lett., 73, 2, pp. 177-184, 2002

[3] E. Guainella, E. Borcoci, M. Katz, P. Neves, M. Curado, F. Andreotti and E. Angori, "WiMAX extension to isolated research data networks", Mobile WiMAX, ed. Wiley, pp. 329-352, 2008

[4] E. Piri, J. Pinola, F. Fitzek, and K. Pentikousis. " $\mathrm{ROHC}$ and Aggregated VoIP over Fixed WiMAX: An Empirical Evaluation”. In Proc. IEEE Symposium on Computers and Communications (ISCC), Marrakech, Morocco, July 2008

[5] F. Andreotti et al., "WEIRD Testbeds with fixed and mobile WiMAX technology for user applications, telemedicine and monitoring of impervious areas", Proc. of TridentCom 2008, March 2008

[6] G.Ash, A. Bader, C. Kappler, D. Oran, "QoS NSLP QSPEC Template", IETF NSIS WG Internet-Draft, April 2008

[7] IEEE 802.16 Working Group, IEEE Standard for Local and Metropolitan Area Networks. Part 16: Air Interface for Fixed Broadband Wireless Access Systems, IEEE Std. 802.16-2004, October 2004

[8] IEEE 802.16 Working Group, IEEE Standard for Local and Metropolitan Area Networks. Part 16: Air Interface for Fixed Broadband Wireless Access Systems. Amendment 2: Physical and Medium Access Control Layer for Combined Fixed and Mobile Operation in Licensed Bands, IEEE Std. 802.16e, December 2005

[9] IEEE 802.21 Working Group, IEEE Draft Standard for Local and Metropolitan Area Networks. Media Independent Handover Services, IEEE P802.21/D9.0, February 2008

[10] I. Harjula, M. Katz, P. Cardamone. "MIMO Techniques for WiMAX Systems in Challenging Radio Channel Conditions". In Proc. of ICT-MobileSummit 2008, Stockholm, Sweden, June 2008

[11] International Electrotechnical Commission. "Precision clock synchronization protocol for networked measurement and control systems". IEC 61588:2004(E), IEEE 1588-2002(E), September 2004
[12] J. Case, M. Fedor, A Simple Network Management Protocol (SNMP), IETF RFC 1157, May 1990

[13] J. Manner. Jugi's Traffic Generator (JTG). http://hoslab.cs.helsinki.fi/savane/projects/jtg

[14] K. Correll. PTP daemon (PTPd). http://ptpd.sourceforge.net

[15] K. Pentikousis, E. Piri, J. Pinola, F. Fitzek, T. Nissilä, and I. Harjula. "Empirical evaluation of VoIP aggregation over a fixed WiMAX testbed". In Proc. Fourth International Conference on Testbeds and Research Infrastructures for the Development of Networks and Communities (TRIDENTCOM), Innsbruck, Austria, March 2008

[16] K. Pentikousis, J. Pinola, E. Piri, and F. Fitzek. "A Measurement Study of Speex VoIP and H.264/AVC Video over IEEE 802.16d and IEEE 802.11 g”. In Proc. Third Workshop on multiMedia Applications over Wireless Networks (MediaWiN), Marrakech, Morocco, July 2008

[17] K. Pentikousis, J. Pinola, E. Piri, and F. Fitzek. "An experimental investigaion of VoIP and video streaming over fixed WiMAX”. In Proc. Fourth International Workshop on Wireless Network Measurements (WiNMee), Berlin, Germany, Mar. 2008

[18] L. Cordeiro, M. Curado, P. Neves, S. Sargento, G. Landi, X. $\mathrm{Fu}$, "Media Independent Handover Network Signalling Layer Protocol (MIH NSLP)", Internet-Draft, IETF, February 2008

[19] O. Grondalen, P. Gronsund, T. Breivik, and P. Engelstad. "Fixed WiMAX field trial measurements and analyses". In Proc. 16th IST Mobile and Wireless Communications Summit, pp. 1-5, July 2007

[20] P. Cardamone, I. Harjula, F. Albiero, M. Katz and L. Mucchi. "WiMAX Channel Model for Mountainous Areas", Accepted for publication at IEEE Vehicular Technology Conference, VTC-2008-fall

[21] R. Hancock et al., "Next Steps in Signalling (NSIS): Framework", RFC 4080, IETF, June 2005

[22] Rosenberg, et al., "SIP: Session Initiation Protocol", RFC 3261, June 2002

[23] WEIRD project web site, http.//www.ist-weird.eu/.

[24] WiMAX Forum, WiMAX End-to-End Network Systems Architecture Stage 2: Architecture Tenets, Reference Model and Reference Points, Release 1.1.0, June 2007

[25] WiMAX Forum, WiMAX End-to-End Network Systems Architecture Stage 3: Detailed Protocols and Procedures, Release 1.1.0, June 2007 